\title{
Western-designed total knee implants mismatch Saudi patient knees
}

\author{
Anatomical bony measurements documented by computed tomography
}

Mohammed L. Al-Otaibi, MD, FRCSC, Ghazi A. Alshumrani, MD, FRCR.

\begin{abstract}

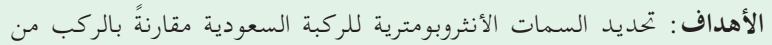

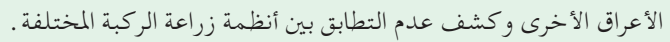

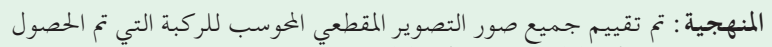

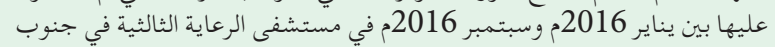

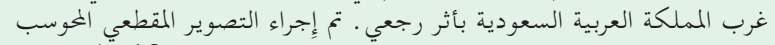

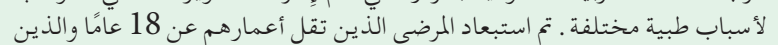

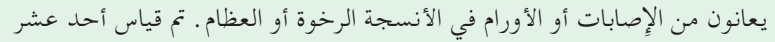

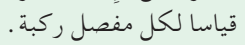

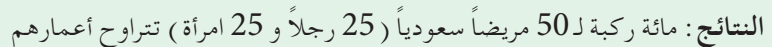

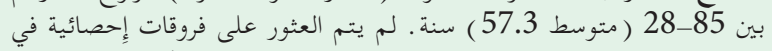

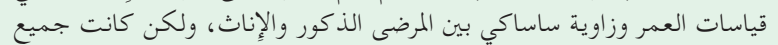

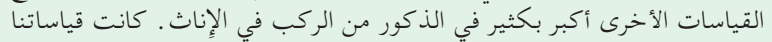

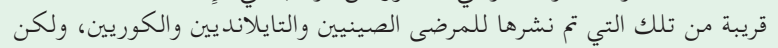

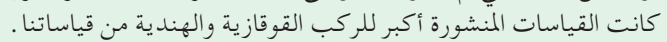

الحلاصة : المرضى السعوديين لديهم سمات مورفولوجية لتشريح عظام الركبة

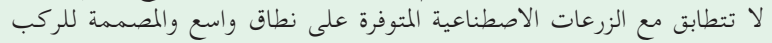

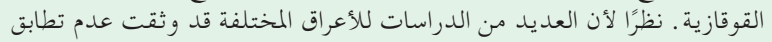

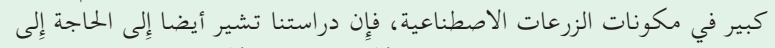

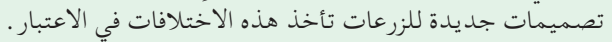

Objectives: To determine the anthropometric features of Saudi knees compared with knees of other ethnicities and reveal mismatches among different knee implant systems.

Methods: All knee computed tomography images obtained between January 2016 and September 2016 for varying medical reasons in a tertiary care hospital in Southwestern Saudi Arabia were retrospectively evaluated. Patients aged $<18$ years, with trauma or soft tissue or bone tumors were excluded. Eleven parameters were measured for each knee joint.

Results: We evaluated 100 knees of 50 Saudi patients (25 men, 25 women) aged 28-85 (mean, 57.3) years. No statistically significant differences were found in age and Sasaki angle measurements between male and female patients. All other parameters were significantly larger in male than female knees. Our measurements were close to those published for Chinese, Thai, and Korean patients; however, published measurements were larger for Caucasian and Indian knees than our measurements.

Conclusion: The Saudi population has morphological features of knee bone anatomy that do not match with the widely available prostheses designed for Caucasian knees. As several studies of different ethnicities have documented considerable prosthesis mismatch; our study further indicates the need for new implant designs that take these variations into account.

Keywords: total knee implant, mismatch, arthroplasty, knee measurements, Saudi knee, CT, ethnic knee, Asian knee

\section{Saudi Med J 2020; Vol. 41 (11): 1227-1233} doi: $10.15537 /$ smj.2020.11.25467

From the Department of Orthopedic Surgery (Al-Otaibi) and from the Department of Radiology (Alshumrani), College of Medicine, King Khalid University, Abha, Kingdom of Saudi Arabia.

Received 11th August 2020. Accepted Gth October 2020.

Address correspondence and reprint request to: Dr. Mohammed L. Al-Otaibi, Associate Professor, Department of Orthopedic Surgery, College of Medicine, King Khalid University, Abha, Kingdom of Saudi Arabia. E-mail:mlalotaibi@kku.edu.sa

ORCID ID: https://orcid.org/0000-0002-5958-9820

$\mathrm{T}$ otal knee implants are designed based on accurate morphologic data of the knee with respect to gender and morphological differences. The more representative the data used in implant design of the general population, the better the expected results. Knee arthroplasty started late in 1968, and over the years, improved surgical techniques and industry have greatly increased its effectiveness and acceptance. Total and 
medial unicompartmental knee arthroplasties represent examples of the successful procedures in medicine with evidence-based favorable outcomes. According to the Agency for Healthcare Research and Quality, more than 600,000 knee replacements are performed annually in the United States, while approximately 35,000 patients undergo knee replacement each year in the United Kingdom. ${ }^{1}$

In the Middle East, including the Kingdom of Saudi Arabia, the annual number of knee replacements has shown a substantial increase over recent years, likely due to the increased survival and high success rates. Knee replacement enables function restoration close to normal, manages pain successfully and increases activities of daily living in patients with knee osteoarthritis. ${ }^{2,3}$ Moreover, technological advances, increased experience of surgeons, as well as improved design of prosthesis components have led to improvement in knee replacement procedures with proper indication. ${ }^{4-6}$

However, differences in knee morphology, which are well established in literature between different ethnicities and Caucasian populations, resonate well with surgeons' observations in Saudi knees in general and in the southwest Saudi region in particular. Mismatch of knee joint replacement implants with the anatomy of Saudi population is observed in the southwestern Saudi region due to small knee sizes in patients undergoing knee replacement. Such mismatch contributes to increased failure rates and complications of knee arthroplasty surgeries. Furthermore, this important topic of implant mismatch and knee measurements in Saudi patients was not adequately investigated previously. Thus, this study aimed to determine the anthropometric features of Saudi knees as part of the Middle East population in comparison with other ethnic and Caucasians knees and to show mismatch among different knee implant systems.

Methods. This retrospective study was approved by the Regional Research Ethics Committee (approval number [ECM\#2020-228] - [HAPO-06-B-001]). The study was conducted in a tertiary care teaching hospital in the Southwestern region of Saudi Arabia.

Disclosure. Authors have no conflict of interests, and the work was not supported or funded by any drug company.
Computed tomography scans of 100 knees of 50 consecutive Saudi patients were included. All knee CT scans obtained from January 1, 2016, through September 20, 2016, were evaluated. Computed tomography scans were performed for variable medical reasons including vascular, joint, bone and soft tissue indications. Patients aged $<18$ years, those with trauma (present or previous), or soft tissue or bone tumors were excluded from the study.

Computed tomography was performed using a dualsource, 128-slice scanner (Somatom Definition Flash, Siemens Healthcare, Germany). The slice thickness of the images was $3 \mathrm{~mm}$. Results were recorded by a consultant radiologist with 10 years of post-board experience. All measurements were performed on the same picture archiving and communication system terminal (Carestream Health, Windsor, CO, USA).

For femoral measurements, an image of maximum dimensions at the level of the femoral epicondyles was chosen, and the following parameters were measured: lateral anteroposterior (LAP) diameter, medial anteroposterior (MAP) diameter, anterior mediolateral length (AML), posterior mediolateral length (PML), and inter-epicondylar (IEC) diameter (Figure 1). For the tibial measurements, an image of the tibial plateau just below the articular cartilage was chosen, and the following parameters were measured: LAP diameter, MAP diameter, anteroposterior (AP) diameter, and tibial transverse diameter (TTD) (Figure 2). For the distance between the medial tibial border and tibial tuberosity (MTT), an image at the center of the tibial tuberosity was chosen, and the maximum straight line was measured from the medial tibial border to the center of the tibial tuberosity (Figure 3). For the patellar angle, an image at the center of the patella was taken to measure the angle according to the Sasaki method (Figure 4).

Statistical analysis. We collected and analyzed data in a Microsoft Excel spreadsheet (Microsoft Corporation, Albuquerque, MM). Data of all male and female patients were analyzed separately to obtain statistics of the measurements and to calculate the P-value of each parameter (Table 1). Published knee measurements from different ethnic groups and available sizes of knee implants were compared with our data and presented in a tabular format (Tables $2 \& 3$ ).

Results. A total of 100 knees of 50 patients (25 men and 25 women) were included. The mean patient age was 57.3 (range, 28-85) years. Eleven parameters were measured for each knee joint with normal distribution of the data in each parameter. No statistically significant differences were found between male knees and female 


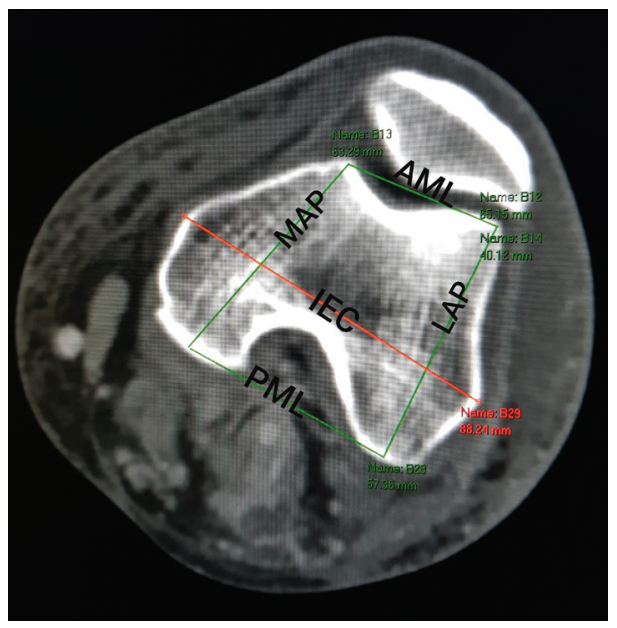

Figure 1 - Femoral measurements. Lateral anteroposterior (LAP) diameter, medial anteroposterior (MAP) diameter, anterior mediolateral length (AML), posterior mediolateral length (PML), and interepicondylar (IEC) diameter.

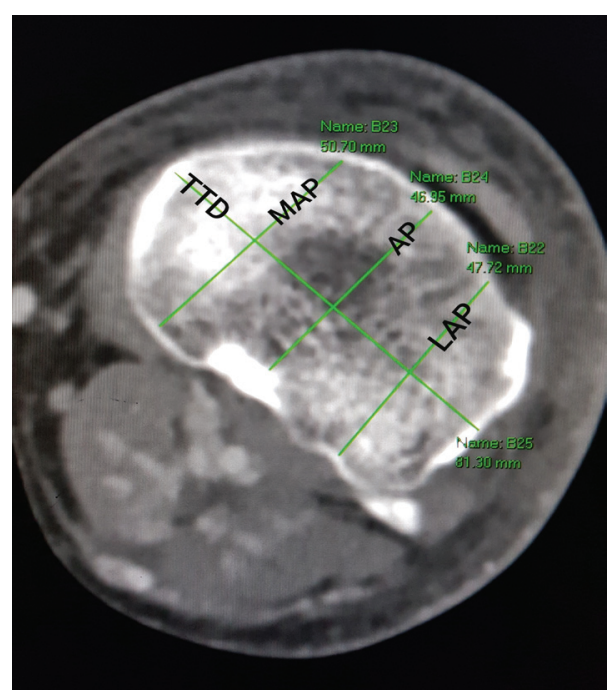

Figure 2 - Tibial measurements. Lateral anteroposterior (LAP) diameter, medial anteroposterior (MAP) diameter, anteroposterior (AP) diameter, and tibial transverse diameter (TTD).

knees in terms of age and Sasaki angle measurements; however, all other parameters were significantly larger in male than in female knees. Although these genderrelated differences were not the focus of this study, they were part of the exploratory data analysis. The results of Saudi knee measurements are summarized in Table 1.

We compared our results with the published knee measurements of different ethnic groups, taking the transverse diameters of the femur and tibia as the

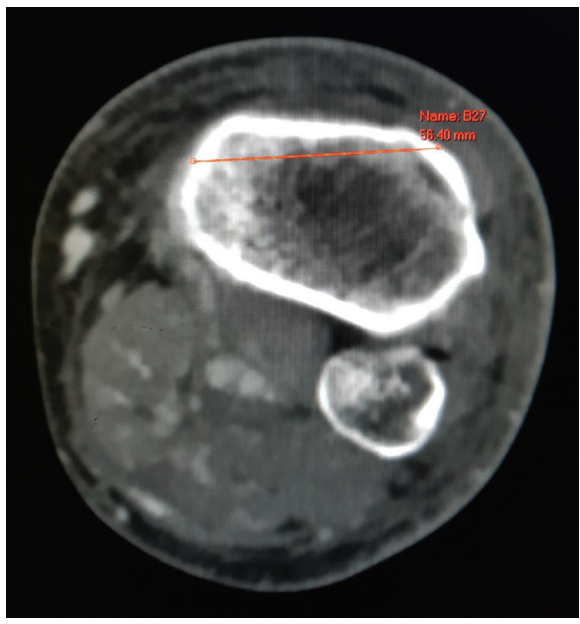

Figure 3 - Measurement of the distance between the medial tibial border and tibial tuberosity.

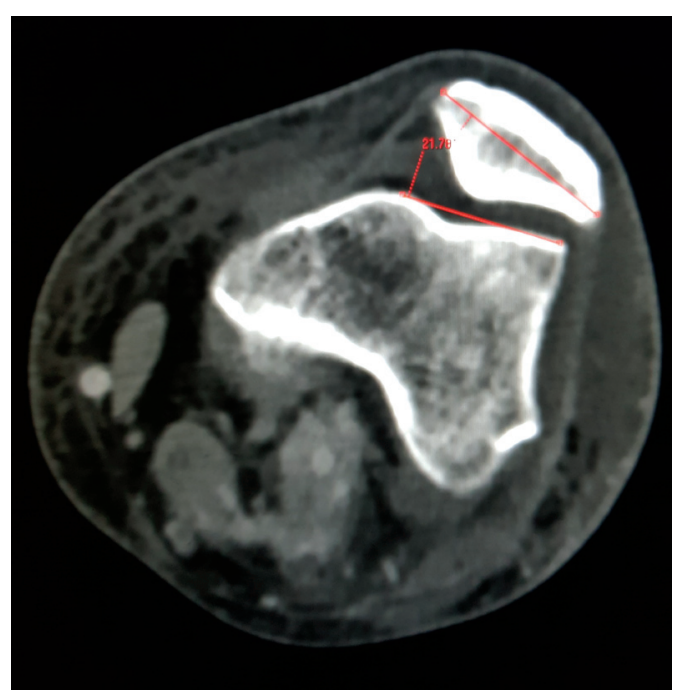

Figure 4 - Patellar angle measurement by the Sasaki method.

reference. Saudi knee measurements were close to the Chinese, Thai, and Korean knee measurements, but smaller than the Indian and Caucasian measurements (Table 2).

We also compared the results of the tibial transverse measurements in our study $(71.4 \mathrm{~mm})$ with the measurements of different available implants. We found that the measurements of Saudi knees are smaller than the available implants, particularly in women (Table 3). Discussion. The results of this study revealed that 
Table 1 - Computed tomography scan measurements of Saudi knees.

\begin{tabular}{lcccccccccc}
\hline Measurements & \multicolumn{3}{c}{ Total $(100$ knees $)$} & \multicolumn{3}{c}{ Men $(50$ knees $)$} & \multicolumn{3}{c}{ Women $(50$ knees $)$} \\
& Min & Max & Mean (SD) & Min & Max & Mean (SD) & Min & Max & Mean (SD) \\
\hline Age & 28.0 & 85.0 & $57.3(18.3)$ & 30.0 & 85 & $55.6(17.6)$ & 28.0 & 83.0 & $59.0(18.9)$ & 0.35 \\
Femur LAP & 52.0 & 68.3 & $59.5(3.7)$ & 52.0 & 68.3 & $61.3(3.7)$ & 53.0 & 62.6 & $57.6(2.5)$ & $<0.001$ \\
Femur MAP & 50.5 & 67.8 & $58.0(4.5)$ & 50.8 & 67.8 & $60.6(4.2)$ & 50.5 & 64.9 & $55.6(3.4)$ & $<0.001$ \\
Femur AML & 24.4 & 41.6 & $33.4(3.3)$ & 29.7 & 41.6 & $35.6(2.8)$ & 24.4 & 34.9 & $31.2(2.3)$ & $<0.001$ \\
Femur PML & 41.0 & 58.4 & $48.5(4.4)$ & 45.0 & 58.4 & $52.0(3.1)$ & 41.0 & 50.9 & $45.1(2.5)$ & $<0.001$ \\
Femur IEC & 65.3 & 88.3 & $76.3(6.0)$ & 65.3 & 88.3 & $80.8(4.7)$ & 66.3 & 80.0 & $71.8(3.0)$ & $<0.001$ \\
Tibia LAP & 31.7 & 49.5 & $40.3(4.0)$ & 34.5 & 49.5 & $42.8(3.6)$ & 31.7 & 42.8 & $37.8(2.6)$ & $<0.001$ \\
Tibia MAP & 35.8 & 54.6 & $45.9(4.2)$ & 40.1 & 54.6 & $48.6(3.4)$ & 35.8 & 49.4 & $43.1(2.8)$ & $<0.001$ \\
Tibia AP & 31.9 & 49.5 & $39.9(4.4)$ & 33.9 & 49.5 & $42.4(3.8)$ & 31.9 & 46.0 & $37.5(3.4)$ & $<0.001$ \\
Tibia TTD & 61.8 & 83.4 & $71.4(5.6)$ & 61.8 & 83.4 & $75.7(4.2)$ & 62.5 & 76.6 & $67.2(3.2)$ & $<0.001$ \\
Tibia MTT & 39.8 & 66.0 & $49.0(5.9)$ & 42.9 & 66 & $52.7(5.4)$ & 39.8 & 54.0 & $45.4(3.6)$ & $<0.001$ \\
Sasaki angle & 9.3 & 33.0 & $19.4(4.2)$ & 10.8 & 28 & $19.3(3.5)$ & 9.3 & 33.0 & $19.6(4.7)$ & 0.76 \\
\hline
\end{tabular}

AML: anterior mediolateral length, AP: anteroposterior diameter, CT: computed tomography, IEC: inter-epicondylar diameter, LAP: lateral anteroposterior diameter, MAP: medial anteroposterior diameter, Min: minimum, MTT: distance between the medial tibial border and tibial tuberosity, PML: posterior mediolateral length, TTD: tibial transverse diameter

Table 2 - Comparison of transverse tibia and femur measurements in Saudi patients and other ethnic groups.

\begin{tabular}{|c|c|c|c|c|}
\hline \multirow{3}{*}{$\begin{array}{l}\text { Ethnic group/References } \\
\text { Saudi (southwestern) }\end{array}$} & \multicolumn{2}{|c|}{ Tibia transverse diameter $(\mathrm{mm})$} & \multicolumn{2}{|c|}{ Femur transverse diameter $(\mathrm{mm})$} \\
\hline & Male & Female & Male & \multirow{2}{*}{$\begin{array}{c}\text { Female } \\
71.8(3.0)\end{array}$} \\
\hline & $75.7 \quad(4.2)$ & $67.2(3.2)$ & $80.8 \quad(4.7)$ & \\
\hline Chinese $^{18}$ & $76.4 \quad(2.8)$ & $68.8 \quad(4.6)$ & $74.4 \quad(2.9)$ & $66.8(3.1)$ \\
\hline Thai ${ }^{19}$ & $74.44 \quad(3.4)$ & $64.95 \quad(3.5)$ & $70.15 \quad(3.9)$ & $59.91(3.8)$ \\
\hline Korean $^{20}$ & $76.1 \quad(4.0)$ & $67.64 \quad(3.1)$ & - & - \\
\hline Indian $^{21}$ & $80.9 \quad(2.6)$ & $72.2(3.6)$ & $83.6 \quad(2.6)$ & $76.3(3.8)$ \\
\hline Caucasian $^{11}$ & $78.7 \quad(5.4)$ & $69.0 \quad(4.2)$ & $86.0 \quad(5.6)$ & $76.4(4.0)$ \\
\hline
\end{tabular}

Table 3 - Saudi knee demonstrating smaller tibial transverse measurements than commercial knee joint knee systems. ${ }^{17}$

\begin{tabular}{lllllll}
\hline Measurements & $\begin{array}{c}\text { Saudi knee } \\
\text { measurements }\end{array}$ & Vanguard & Genesis 2 & $\begin{array}{c}\text { Persona } \\
\text { narrow }\end{array}$ & $\begin{array}{c}\text { Persona } \\
\text { standard }\end{array}$ & $\begin{array}{c}\text { Atune } \\
\text { standard }\end{array}$ \\
\hline $\begin{array}{l}\text { Tibial component } \\
\text { medial to lateral }(\mathrm{mm})\end{array}$ & $71.4(5.6)$ & $75(10.9)$ & $73(8.5)$ & $72(10.4)$ & $72(10.4)$ & $73(9.1)$ \\
\hline
\end{tabular}


Saudi knee measurements were smaller than that of some ethnic groups and the available knee implants. Relatively short stature, small bone structures and high prevalence of varus deformity are all surgical observations in Southwestern Saudi population when compared with Caucasians. Attempts to study different factors playing a role in knee replacement success revealed that factors related to patient, surgeon, surgical techniques, and implant design play a role in total knee replacement outcomes. Knee replacement procedures carry a higher success rate when performed by board-certified welltrained surgeons applying the most up-to-date surgical techniques and using latest implant designs approved by the Food and Drug Administration. ${ }^{7}$ A considerable degree of implant mismatch with knees of Saudi patients have been reported, similar to mismatches reported in other ethnic groups. ${ }^{8}$ For over 50 years, the standard surgical procedure for advanced knee osteoarthritis has been total knee replacement with solid evidencebased results. ${ }^{9}$ Nearly 2.9 million knee replacements have been performed worldwide. ${ }^{10}$ In the recent years, there has been a substantial increase in the survival rate of up to $95 \%$ due to improved surgical technique, implant design, and patients' acceptance to undergo the procedure. ${ }^{11}$ However, early and late complications are still major concerns, but are well looked after in terms of measures to prevent their development and wellestablished treatment protocols. Surgeons worldwide have observed that total knee replacement procedures are challenged by the prosthesis mismatch related to variations in the anatomy of different ethnic groups. As knee replacement implants are mostly manufactured in accordance with the knee anatomy of the Caucasian populations, considerable mismatch arises when implanted to non-Caucasian knees. ${ }^{12}$ The small sizes of the available implant can be close to the desired implant size of some ethnic groups, but may still be bigger than the needed size for many patients. Issues of size have been addressed to some extent by the development of new implants accommodating small sizes and considering anatomical differences. The surgical technique of knee replacement uses certain bony references and landmarks, which have been documented to vary in different ethnic groups. ${ }^{13}$ Long-standing varus tibial deformity, which appears to be common in this part of the world, may result in late proximal tibial insufficiency fracture that may be masked by the arthritic knee pain. Therefore, such insufficiency fractures may only be discovered intraoperatively during knee replacement, which will be another challenge added to the intraoperative difficulty caused by implant mismatch. ${ }^{14}$ Here in our study, we have provided a reference measurement of the anatomical axis of the tibia (measured from the tibial tuberosity to the medial tibial border).

In Saudi Arabia, a small number of knee replacements were performed in the 1990 s. ${ }^{15}$ Over the last 30 years, there has been a noticeable increase in knee replacement procedures due to the presence of many well-equipped specialized centers with well-trained personnel. In general, Saudi knees have anatomical variations when compared with Caucasian populations but resemble the Asian knees to certain extent. Furthermore, the Southwestern region of Saudi Arabia has drawn the attention of joint reconstruction surgeons due to the small sizes of the implanted components in knee replacement.

Southwestern Saudi Arabia is a geographically different region, and its population has an increased prevalence of obesity. ${ }^{16}$ In reference to the anatomical variation of ethnic knees, multiple studies have reported current mismatch in some designs of knee replacement implants. The width of the distal femur is smaller than that in Caucasian populations. The recently available new implant design with narrow femoral components have addressed this problem to some extent in Saudi patients undergoing knee replacement. ${ }^{17}$ Our study indicated that the available prostheses that are mostly used in Caucasian patients, with the exception of the recently available anatomical knee prosthesis, may be larger than ideal for Saudi patients. ${ }^{17}$

With regard to the anatomical tibial axis in the Caucasian population, several studies have revealed that it is located anteromedial to the center of the tibial plateau, whereas in several other studies of ethnic knees in Asia and certainly in our study group, the entry point would be just lateral to the center point. Component mismatch in our patients follows what is well documented in recent literature about Asian knees. The mean tibial and femoral knee transverse diameters in our study were close to the measurements published for Chinese, Thai, and Koreans. ${ }^{18-20}$ However, measurements in Caucasian and Indian knees were larger than our measurements (Table 2). ${ }^{11,21}$

The morphology of prostheses used among the Southwestern Saudi population was compared with that of total knee prostheses currently used worldwide, and it was found that the femoral aspect ratio (mediolateral/ anteroposterior) of these prostheses were not suitable for Saudi patients, based on local surgeon observations. Our femoral measurements were smaller than those in Caucasian and Indian knees. ${ }^{22}$ Patellofemoral problems are common among the Saudi population, partly due to the lack of exercise. Despite symptomatic patellofemoral arthritis, Saudi patients present late for 
knee replacement, that is, only when their daily life activities are greatly compromised. We believe that patient acceptance of knee replacement surgery may be improved by using implants that match the anatomy to prevent postoperative pain caused by implant mismatch, for example, overhanging of the tibial tray and iliotibial band impingement. ${ }^{23,24}$

Women represent the majority of patients undergoing knee replacement in Saudi Arabia. Internationally, women have shown a tendency to be of shorter stature and relatively overweight. ${ }^{25}$ Men represent less challenge in implant mismatch than women. The Saudi culture contributes to some of the changes that may influence knee replacement outcome, such as sitting on the floor in kneeling position, which may lead to varus deformity and proximal tibial torsion. ${ }^{26}$ Measurements from CT images in this study documented that Saudi knee is smaller than the Caucasian knee. Previously, studies have reported that the knees of different ethnic groups are generally smaller than Caucasian knees. ${ }^{27,28}$ Our finding confirms that Saudi knees are smaller than Caucasian knees and is consistent with the results of other ethnic studies on knees. Such studies documented considerable mismatch when some of the available knee prostheses were implanted in Saudi patients (Table 3). Special consideration in designing prosthesis components for knee replacement started worldwide when implant sizes required for different ethnic groups were questioned and poor results were reported. Moreover, early reports about implant design have led to maximizing tibial coverage and eliminating asymmetry in implant design. ${ }^{29}$ Insufficiency fractures are more common in Saudis following knee replacement due to osteoporosis, overweight, and gross varus deformity. ${ }^{30}$

In Asian countries, Chinese knees have an average tibial coronal and tibial sagittal measurements of $73.0(4.6) \mathrm{mm}$ and $48.8(3.4) \mathrm{mm}$, respectively. Men have larger coronal and sagittal measurements than women. Male knees have wider dimensions than female knees in the coronal plane under a given sagittal dimension. ${ }^{22}$ Studies showed that the femoral coronal and sagittal dimensions were strongly correlated with the tibial coronal dimension. As the tibial coronal dimension increased, the femoral coronal dimension and femoral sagittal dimension also increased. Studies found that Chinese women have generally narrower intercondylar distance than men. ${ }^{31,32}$ Recently available knee replacement implants, with standard and narrow femoral components for each size, efficiently address the mediolateral dimensions without overhang. Similarly, the $2 \mathrm{~mm}$ differences in anteroposterior dimensions between sizes, achieves optimal joint balance with minimal bone resection. This was not possible in case of older knee systems available in the market, which were suitable for western patients where smaller anatomy is not an issue.

The surgical technique of knee replacement is dependent on bony landmarks, and one crucial step is to establish the anatomical axis of the tibia. Varus deformity is less common in the West than in the East. Western population studies have shown that the axis of the tibial shaft is located anteromedial to the center of the tibial plateau. ${ }^{32}$ The anatomical axis of the tibia exits lateral to the center point of the tibial plateau in most other ethnic knees. ${ }^{23}$ Caucasian knees have low posterior slope, whereas Saudi and Asian knees have increasing posterior tibial plateau slope. ${ }^{33}$ This could possibly be attributed to squatting, kneeling, and deep flexion which are not common in Western daily activities. Multiple studies in Chinese, Japanese, Korean, and Indian populations found that the mediolateral diameter of the distal femur is smaller than their Caucasian counterparts with the same anteroposterior diameter. ${ }^{34}$

Recent anthropometric studies concluded that the current design of total knee replacement does not consider ethnic differences. Most of the commercially available knee replacement prostheses are designed according to the measurement data of Caucasian knees, which lead to the current component mismatch in Asians and Saudi Arabian patients. ${ }^{22,34}$

Study limitations. The main limitations of our study are its retrospective design and the small sample size which may limit generalization of the results on Saudi population. Larger national studies in different regions of Saudi Arabia are needed.

In conclusion, the Saudi population shows certain morphological features of the knee bone anatomy that do not match with the widely available Western-designed knee prostheses. Several studies have documented increased knee implant mismatch, which is also shown in our study; therefore, such considerable prosthesis component mismatch indicates the need for new implant design that takes these variations into account. Further studies evaluating considerable mismatch in Saudi knees will contribute to the understanding of persistent postoperative pain and explain the mode of knee replacement failure. Studies are also needed to investigate whether newly available prosthesis designs reduce such mismatch. Women are universally found to have a significantly smaller knee size than men in southwestern Saudi Arabia, and this should be considered during the design and manufacture of prosthetic knee implants in Saudi Arabia. 
Acknowledgment. We would like to thank Editage (www. editage.com) for English language editing.

\section{References}

1. Moran CG, Horton TC. Total knee replacement: the joint of the decade. A successful operation, for which there's a large unmet need. BMJ 2000; 320: 820.

2. Andriacchi TP. Functional analysis of pre and post-knee surgery: total knee arthroplasty and ACL reconstruction. J Biomech Eng 1993; 115: 575-581.

3. Myles CM, Rowe PJ, Walker CR, Nutton RW. Knee joint functional range of movement prior to and following total knee arthroplasty measured using flexible electrogoniometry. Gait Posture 2002; 16: 46-54.

4. Rand JA, Ilstrup DM. Survivorship analysis of total knee arthroplasty. Cumulative rates of survival of 9200 total knee arthroplasties. J Bone Joint Surg Am 1991; 73: 397-409.

5. Ranawat CS, Luessenhop CP, Rodriguez JA. The press-fit condylar modular total knee system: four-to-six year results with a posterior-cruciate-substituting design. J Bone Joint Surg Am 1997; 79: 342-348.

6. Rasquinha VJ, Ranawat CS, Cervieri CL, Rodriguez JA. The press-fit condylar modular total knee system with a posterior cruciate-substituting design. A concise follow-up of a previous report. J Bone Joint Surg Am 2006; 88: 1006-1010.

7. Zulkifly AH, Masbah O, Ruslan G. Total knee replacement: 12 years retrospective review and experience. Malays Orthop J 2011; 5: 34-39.

8. Clarke HD, Hentz JG. Restoration of femoral anatomy in TKA with unisex and gender-specific components. Clin Orthop Relat Res 2008; 466: 2711-2716.

9. Ranawat CS. History of total knee replacement. J South Orthop Assoc 2002; 11: 218-226.

10. Kurtz SM, Ong KL, Lau E, Widmer M, Maravic M, GómezBarrenaE, et al. International survey of primary and revision total knee replacement. Int Orthop 2011; 35: 1783-1789.

11. Mahfouz M, Abdel Fatah EE, Bowers LS, Scuderi G. Threedimensional morphology of the knee reveals ethnic differences. Clin Orthop Relat Res 2012; 470: 172-185.

12. Hitt K, Shurman JR 2nd, Greene K, McCarthy J, Moskal J, Hoeman T, et al. Anthropometric measurements of the human knee: correlation to the sizing of current knee arthroplasty systems. J Bone Joint Surg Am 2003; 85-A Suppl 4: 115-122.

13. Moreland JR, Bassett LW, Hanker GJ. Radiographic analysis of the axial alignment of the lower extremity. J Bone Joint Surg Am 1987; 69: 745-749.

14. Rashid RH, Zubairi AJ, Umer M, Hashmi PM, Lakdawala RH. Management of stress fractures of the proximal tibia in patients with advance knee osteoarthritis. A case series. Acta Orthop Belg 2018; 84: 436-442.

15. Ahlberg A. Knee joint replacement in Saudi Arabia - present and future. Ann Saudi Med 1994; 14: 281-282.

16. Al-Qahtani AM. Prevalence and predictors of obesity and overweight among adults visiting primary care settings in the southwestern region, Saudi Arabia. BioMed Res Int 2019; 8073057: 5 .

17. Budhiparama NC, Lumban-GaolI, Ifran NN, de Groot PC, Utomo DN, Nelissen RGHH. Mismatched knee implants in Indonesian and Dutch patients: a need for increasing the size. Knee Surg Sports Traumatol Arthrosc 2020; Mar: DOI: 10.1007/s00167-020-05914-9.
18. Yue B, Varadarajan, KM Ai S, Tang T, Rubash HE, Li G. Gender differences in the knees of Chinese population. Knee Surg Sports Traumatol Arthrosc 2011; 19: 80-88.

19. Chaichankul C, Tanavalee A, Itiravivong P. Anthropometric measurements of knee joints in Thai population: correlation to the sizing of current knee prostheses. Knee 2011; 18: 5-10.

20. Kwak DS, Surendran S, Pengatteeri YH, Park SE, Choi KN, Gopinathan P, et al. Morphometry of the proximal tibia to design the tibial component of total knee arthroplasty for the Korean population. Knee 2007; 14: 295-300.

21. Vaidya SV, Ranawat CS, Aroojis A, Laud NS. Anthropometric measurements to design total knee prostheses for the Indian population. J Arthroplasty 2000; (1): 79-85.

22. Hosseinzadeh HRS, Tarabichi S, Shahi AS, Yeganeh MH, Saleh UH, Kazemian GR, et al. In: Kinov P, editor, Special Considerations in Asian Knee Arthroplasty. Chapter. Intech Creative Commons Licence. [cited 2013]. Available from: https://www.doi.org/10.5772/53595

23. Kwok KB, Fan JCH, Hung YW, Chan APH, Bernard TWC, CLau LCM. Iliotibial band impingement by overhanging tibial tray: a pain generator after total knee replacement treatable without revision. J Orthop Trauma Rehabilitation 2018; 25: 54-57.

24. Abram SGF, Marsh AG, Nicol F, Brydone AS, Mohammed A, Spencer SJ. The impact of tibial component overhang on outcome scores and pain in total knee replacement. Orthop Proc 2018; 96-B.

25. Leung YY, Allen JC Jr, Novianiy M. Ang LW, Wang R, Yuan $\mathrm{JM}$, et al. Association between body mass index and risk of total knee replacement, the Singapore Chinese Health Study. Osteoarthr Cartil 2015; 23; 41-47.

26. Cooke DT, Harrison L, Khan B, Scudamore AM, Chaudhary A. Analysis of limb alignment in the pathogenesis of osteoarthritis: a comparison of Saudi Arabian and Canadian cases. Rheumatol Int 2002; 22: 160-164.

27. Kim DK, Seo MC, Song SJ, Kim KI. Are Korean patients different from other ethnic groups in total knee arthroplasty? Knee Surg Relat Res 2015; 27: 199-206.

28. Cheng FB, Ji XF, Lai Y, Feng JC, Zheng WX, Sun YF, et al. Three-dimensional morphometry of the knee to design the total knee arthroplasty for Chinese population. Knee 2009; 16: 341-347.

29. Westrich GH, Haas SB, Insall JN, Frachie A. Resection specimen analysis of proximal tibial anatomy based on 100 total knee arthroplasty specimens. J Arthroplasty 1995; 10 : 47-51.

30. Sadat-Ali M, Al-Habdan IM, Al-Turki HA, Azama MQ. An epidemiological analysis of the incidence of osteoporosis and osteoporosis-related fractures among the Saudi Arabian population. Ann Saudi Med 2012; 32: 637-641.

31. Hicks CA, Noble P, Tullos H. The anatomy of the tibial intramedullary canal. Clin Orthop Relat Res 1995; 321: 111-116.

32. Tang Q, Zhou Y, Yang D, Xu H, Liu Q. The offset of the tibial shaft from the tibial plateau in Chinese people. J Bone Joint Surg Am 2010; 92: 1981-1987.

33. Kapandji IA. The knee ligaments as determinants of trochleocondylar profile. Med Biol Illus 1967; 17: 26-32.

34. Otoukesh B, Boddouhi B, Kaghazian P, Hatami N, Otoukesh M, Ghaemi M, et al. Evaluation of 3-dimensional profile of Asian knee. Ann Med Health Sci Res 2017; 7: 61-66. 